\title{
ASSOCIAÇÃO ENTRE ISOENZIMAS E MATÉRIA SECA EM BATATA SILVESTRE ${ }^{1}$
}

\author{
BEATRIZ HELENA GOMES ROCHA², ELIANE AUGUSTIN ${ }^{3}$, \\ JOÃO BAPTISTA DA SILVA ${ }^{4}$ e ARIONE DA SILVA PEREIRA ${ }^{5}$
}

\begin{abstract}
RESUMO - Clones de batata silvestre (Solanum spp.) foram analisados quanto aos teores de matéria seca em tubérculos e isoenzimas de aspartato transaminase e isocitrato desidrogenase em folhas, com o objetivo de identificar associação entre estas características. Os resultados obtidos em uma amostra de 50 clones indicam grande variabilidade do teor de matéria seca. Clones de $S$. commersonii malmeanum apresentaram maior teor que os de $S$. commersonii commersonii, S. chacoense muelleri e de outros cuja espécie não foi identificada. Os padrões isoenzimáticos de folhas de 38 clones da amostra mostraram 15 variantes eletroforéticas de aspartato transaminase e sete de isocitrato desidrogenase. Comparações efetuadas através de tabelas de contingência 2 × 2 , usando o teste de $\chi^{2}$, com correção para continuidade, permitiram concluir que existe associação significativa entre o teor de matéria seca e as bandas de mobilidade relativa 1,00 de aspartato transaminase e de isocitrato desidrogenase.
\end{abstract}

Termos para indexação: Solanum, aspartato transaminase, isocitrato desidrogenase, melhoramento de plantas.

\section{ISOENZYMES AND DRY MATTER CONTENT IN WILD POTATO}

\begin{abstract}
Wild potato clones (Solanum spp.) were analysed for tuber dry matter content, leaf aspartate transaminase and leaf isocitrate dehydrogenase isoenzymes in order to identify association between these characteristics. The results indicate that there is great variability for dry matter content among 50 clones analysed. S. commersonii malmeanum clones had higher dry matter levels than S. commersonii commersonii, S. chacoense muelleri and the clones whose species were not identified. Fifteen aspartate transaminase and seven isocitrate dehydrogenase bands were observed in the gels of 38 clones. Tests of $\chi^{2}$, using $2 \times 2$ contingency table, revealed a significant association between dry matter content and the bands of relative mobility 1.00 of both aspartate transaminase and isocitrate dehydrogenase isoenzymes.
\end{abstract}

Index terms: Solanum, aspartate transaminase, isocitrate dehydrogenase, plant breeding.

${ }^{1}$ Aceito para publicação em 9 de fevereiro de 2000.

${ }^{2}$ Eng. Agrôn., M.Sc., doutorando, Dep. de Fitotecnia, Universidade Federal de Pelotas (UFPEL), Caixa Postal 354, CEP 96001-970 Pelotas, RS. Bolsista do CNPq. E-mail: biagr@terra.com.br

${ }^{3}$ Eng. Agrôn., Dr., Embrapa-Centro de Pesquisa Agropecuária de Clima Temperado (CPACT), Caixa Postal 403, CEP 96001-970 Pelotas, RS. E-mail: augustin@cpact.embrapa.br

${ }^{4}$ Eng. Agrôn., Dr., Prof. Titular, Dep. de Matemática, Estatística e Computação, UFPEL. Bolsista do CNPq. E-mail: jbsilva@ufpel.tche.br

${ }^{5}$ Eng. Agrôn., Ph.D., Embrapa-CPACT. Bolsista do CNPq. E-mail: arione@cpact.embrapa.br

\section{INTRODUÇÃO}

Não existem cultivares nacionais de batata apropriadas ao processamento industrial, nem tampouco cultivares estrangeiras adaptadas às condições de cultivo do Sul do Brasil (Costa \& Pereira, 1996). Cultivares para processamento na forma de palitos devem conter teores de sólidos totais acima de $20 \%$, pois ao absorver pouca quantidade de óleo durante a fritura, são mais econômicas e resultam em produtos processados de melhor qualidade (Almeida et al., 1983). Dessa forma, no melhoramento para o processamento industrial visando atingir teores mais 
elevados de matéria seca tem-se tentado utilizar espécies diplóides silvestres e primitivas (Mendoza, 1990). Espécies da série Commersoniana, originárias da Argentina, Bolívia, Brasil, Paraguai e Uruguai, juntamente com espécies das séries Demissa e Longipedicellata do México, são de especial interesse ao melhoramento, graças à superioridade dos teores de matéria seca com conteúdo de amido acima de $33,40 \%$ em Solanum chacoense e $27,7 \%$ em S. commersonii (Hawkes \& Hjerting, 1969).

No melhoramento genético vegetal, as isoenzimas têm sido utilizadas com diferentes finalidades, como para avaliação de germoplasma, detecção de ligação gênica com caracteres mono e poligênicos, e seleção indireta de caracteres agronômicos (Soller \& PlotkinHazan, 1977; Tanksley et al., 1981, 1982; MedinaFilho, 1983). Vários autores referem-se a polimorfismos enzimáticos em diferentes espécies de Solanum (Oliver \& Martinez-Zapater, 1985; Douches et al., 1989; Douches \& Ludlam, 1991).

O objetivo deste trabalho foi identificar a associação existente entre teores de matéria seca e as isoenzimas de aspartato transaminase e de isocitrato desidrogenase em folhas de tubérculos de batata silvestre.

\section{MATERIAL E MÉTODOS}

Foram analisados, em 1996, nos laboratórios de Fisiologia Vegetal e de Eletroforese da Embrapa-Centro de Pesquisa Agropecuária de Clima Temperado, teores de matéria seca e padrões isoenzimáticos de aspartato transaminase e isocitrato desidrogenase de, respectivamente, 50 e 38 clones de batata silvestre das subespécies S. commersonii malmeanum, S. commersonii commersonii, S. chacoense muelleri e Solanum spp. não identificadas, coletadas de 1986 a 1990, no Rio Grande do Sul e Santa Catarina (Tabela 1), e mantidos no banco de germoplasma de hortaliças dessa unidade.

A análise da matéria seca foi realizada com amostras de 2 a $5 \mathrm{~g}$ de tubérculos. Estes foram colocados em estufa, com circulação de ar à temperatura de, aproximadamente, $60^{\circ} \mathrm{C}$, onde permaneceram 13 a 14 dias, quando atingiram peso constante. O delineamento experimental usado foi o inteiramente casualizado, com quatro repetições. Após a análise da variância, utilizou-se o teste de Duncan para comparação das médias.
$\mathrm{Na}$ análise de isoenzimas foi utilizada eletroforese horizontal em gel de poliacrilamida nas concentrações de $6 \%$ e $5 \%$. Para aspartato transaminase, utilizou-se a solução-tampão de Scandalios (1969) e o sistema de coloração de Ayala et al. (1972). Para a isocitrato desidrogenase, utilizaram-se os tampões de Nichols \& Ruddle (1973) modificado (pH 6,5) e de Shields et al. (1983), e o sistema de coloração de Vallejos (1983).

Nas análises de aspartato transaminase, foram maceradas amostras de $10 \mathrm{mg}$, coletadas na terceira ou quarta folha, antes da floração das plantas. Nas análises de isocitrato desidrogenase, $10 \mathrm{mg}$ de amostras, coletadas na quinta ou sexta folha de plantas em plena floração, foram maceradas em $0,01 \mathrm{~mL}$ de tampão citrato-histidina pH 6,5, acrescido de $0,15 \%$ de 2-mercaptoetanol.

Amostras do clone 186 foram usadas como controle. A mobilidade relativa de cada banda foi calculada em relação a uma banda de referência deste clone, à qual foi atribuído valor relativo igual a 1 .

As associações entre clones com maiores ou menores teores de matéria seca e isoenzimas foram avaliadas a partir de tabelas de contingência $2 \times 2$, por meio do teste de $\chi^{2}$, com correção para continuidade (Yates), para os níveis de probabilidade de $5 \%$ e $1 \%$.

\section{RESULTADOS E DISCUSSÃO}

A análise da variância do conteúdo de matéria seca dos 50 clones silvestres mostrou que as diferenças entre os genótipos foram altamente significativas (Tabela 1). Os teores médios obtidos, com variação entre $40,08 \%$ e $24,05 \%$ (Tabela 2), são considerados altos, quando comparados com outros citados na literatura. Akeley et al. (1968) verificaram que o teor de sólidos totais da cultivar Lenape, em diferentes locais, foi de, aproximadamente, $22,9 \%$, enquanto

TABELA 1. Análise da variância do conteúdo de matéria seca dos 50 clones silvestres de Solanum spp.

\begin{tabular}{lrrrr}
\hline Causa da variação & G. L. & S.Q. & Q.M & Valor F \\
\hline Clones & 49 & 953,67 & 19,46 & $16,30^{* * *}$ \\
Resíduo & 150 & 179,13 & 1,19 & \\
Total & 199 & 1132,80 & & \\
\hline C. V. (\%) & \multicolumn{5}{c}{3,17} \\
Média geral & 34,42 & & \\
\hline
\end{tabular}

** Significativo a $1 \%$ de probabilidade. 
TABELA 2. Procedência, ano de coleta e porcentagens médias de matéria seca de clones de Solanum commersonii commersonii (SCC), S. commersonii malmeanum (SCM), S. chacoense muelleri (SChM) e de subespécie não-identificada (NI) ${ }^{1}$.

\begin{tabular}{|c|c|c|c|c|}
\hline Clone & Grupo & Procedência & Ano & Porcentagem de matéria seca \\
\hline 113 & SCC & Santo Ângelo, RS & 1988 & $40,08 \mathrm{a}$ \\
\hline 60 & $\mathrm{SCM}^{2}$ & Porto Lucena, RS & 1987 & $37,41 \mathrm{~b}$ \\
\hline 118 & SCC & Torres, RS & 1988 & $36,55 \mathrm{bc}$ \\
\hline 08 & $\mathrm{SCM}$ & Augusto Pestano, RS & 1986 & $36,09 \mathrm{bcd}$ \\
\hline 57 & $\mathrm{SCM}^{2}$ & Santo Ângelo, RS & 1987 & $35,74 \mathrm{bcde}$ \\
\hline 69 & SCM & Tenente Portela, RS & 1987 & $35,32 \mathrm{bcdef}$ \\
\hline 176 & SCC & São Borja, RS & 1989 & $35,29 \mathrm{bcdef}$ \\
\hline 108 & $\mathrm{SCM}$ & Sarandi, RS & 1988 & $35,28 \mathrm{bcdef}$ \\
\hline 56 & SCM & Santo Ângelo, RS & 1987 & $35,21 \mathrm{bcdef}$ \\
\hline 62 & SCM & Porto Xavier, RS & 1987 & $35,06 \mathrm{bcdefg}$ \\
\hline 09 & $\mathrm{SCC}^{2}$ & Ijuí, RS & 1986 & $34,98 \mathrm{bcdefg}$ \\
\hline 67 & SCM & Crissiumal, RS & 1987 & $34,77 \mathrm{bcdefg}$ \\
\hline 63 & $\mathrm{SCM}^{2}$ & Santo Cristo, RS & 1987 & 34,68 bcdefg \\
\hline 07 & SCC & Uruguai & 1986 & $34,55 \mathrm{bcdefgh}$ \\
\hline 55 & $\mathrm{SCM}$ & Augusto Pestano, RS & 1987 & 34,48 bcdefgh \\
\hline 65 & $\mathrm{SCM}$ & Horizontina, RS & 1987 & 34,39 bcdefgh \\
\hline 53 & SCC & Pelotas, RS & 1987 & 34,39 bcdefgh \\
\hline 120 & SCC & Morro dos Conventos, SC & 1988 & 34,26cdefghi \\
\hline 02 & $\mathrm{SCM}$ & São Miguel das Missões, RS & 1986 & 33,51cdefghij \\
\hline 230 & $\mathrm{SCC}^{2}$ & Santiago, RS & 1990 & 33,48cdefghij \\
\hline 201 & $\mathrm{NI}^{2}$ & Alegrete, RS & 1990 & 33,16defghijk \\
\hline 168 & SCC & São José do Norte, RS & 1989 & 32,95efghijkl \\
\hline 100 & $\mathrm{SCC}^{2}$ & Santa Vitória do Palmar, RS & 1988 & 32,69efghijkl \\
\hline 64 & SCM & Tuparendi, RS & 1987 & 32,51 fghijkl \\
\hline 224 & $\mathrm{NI}^{2}$ & São Borja, RS & 1990 & 32,51fghijkl \\
\hline 196 & SCC & Quarai, RS & 1990 & 32,43fghijkl \\
\hline 208 & $\mathrm{NI}^{2}$ & São Borja, RS & 1990 & 32,01 ghijklm \\
\hline 222 & NI & Itaqui, RS & 1990 & 32,00ghijklm \\
\hline 142 & SCC & Santa Cruz do Sul, RS & 1988 & 31,99ghijklm \\
\hline 119 & SCC & Morro dos Conventos, SC & 1988 & 31,56hijklm \\
\hline 199 & NI & Uruguaiana, RS & 1990 & 31,33ijklmn \\
\hline 25 & SCC & Pelotas, RS & 1986 & 31,27ijklmno \\
\hline 139 & SCC & Candelária, RS & 1988 & 31,26ijklmno \\
\hline 143 & SCC & São Francisco de Paula, RS & 1988 & 30,67jklmnop \\
\hline 117 & SCC & Torres, RS & 1988 & 30,61jklmnop \\
\hline 141 & SCC & Vera Cruz, RS & 1988 & 30,55jklmnop \\
\hline 192 & $\mathrm{SCC}^{2}$ & Santana do Livramento, RS & 1990 & 30,49jklmnop \\
\hline 109 & SCM & Iraí, RS & 1988 & 30,26klmnopq \\
\hline 36 & SCC & Rio Grande, RS & 1986 & 30,061mnopq \\
\hline 131 & SCC & Soledade, RS & 1988 & $29,27 \mathrm{mnopqr}$ \\
\hline 71 & SCC & Santa Maria, RS & 1987 & 28,50nopqr \\
\hline 166 & $\mathrm{SCC}$ & Rio Grande, RS & 1989 & 28,41 opqr \\
\hline 78 & SCC & São Lourenço do Sul, RS & 1987 & $27,97 \mathrm{pqrs}$ \\
\hline 54 & SCC & Lajeado, RS & 1987 & $27,94 \mathrm{pqrs}$ \\
\hline 250 & $\mathrm{NI}^{2}$ & Erechim, RS & 1991 & $27,48 \mathrm{qrs}$ \\
\hline 200 & NI & Alegrete, RS & 1990 & 26,60 rst \\
\hline 158 & SCC & Camaquã, RS & 1989 & $25,65 \mathrm{st}$ \\
\hline 150 & SCC & Lagoa Vermelha, RS & 1988 & $24,15 t$ \\
\hline 68 & SChM & Três Passos, RS & 1987 & $24,12 \mathrm{t}$ \\
\hline 164 & $\mathrm{SCC}^{2}$ & Rio Grande, RS & 1989 & $24,05 \mathrm{t}$ \\
\hline
\end{tabular}

1 As médias seguidas pela mesma letra não diferem entre si pelo teste de Duncan $(\alpha=0,05)$.

2 Clones não submetidos à análise isoenzimática. 
os de Katahdin e Kennebec foram de 17,7\% e $18,4 \%$, respectivamente. Dois híbridos de S. tuberosum x S. stoloniferum, contendo altos teores de sólidos (23\% e 26,9\%) foram comparados, por Pope et al. (1971), com as cultivares comerciais Russet Burbank (22,9\%), usadas como controle para flocos de batata e fritas à francesa, e Kennebec $(20,1 \%)$, como padrão para "chips". Os produtos destes materiais geralmente apresentaram melhor cor e rendimento do que as cultivadas. Em cinco cultivares de batatas americanas, foram encontrados valores que oscilaram entre $18 \%$ e $21,5 \%$ (Gould, $1988)$. Alto teor de matéria seca $(22,9 \%)$ foi observado na cultivar nacional Catucha (Silva et al., 1996), que possui uma espécie silvestre em sua genealogia.

A análise da variância e comparações entre as médias da porcentagem de matéria seca, por espécies, indicaram diferenças significativas (Tabelas 3

TABELA 3. Análise da variação do conteúdo de matéria seca dos grupos Solanum commersonii malmeanum, $S$. commersonii commersonii e espécie não-identificada.

\begin{tabular}{lcrrr}
\hline Causa da variação & G. L. & S. Q. & Q. M. & Valor F \\
\hline Clones & 2 & 120,56 & 60,28 & $6,39 * *$ \\
Resíduo & 46 & 433,89 & 9,43 & \\
Total & 48 & 554,45 & & \\
\hline C.V. (\%) & 9,55 & & \\
Média geral & 32,16 \\
** Significativo a 1\% de probabilidade.
\end{tabular}

TABELA 4. Porcentagens médias de matéria seca dos grupos Solanum commersonii malmeanum (SCM), S. commersonii commersonii (SCC) e de espécie não-identificada (NI) ${ }^{1}$.

\begin{tabular}{ccc}
\hline Grupo silvestre & $\begin{array}{c}\text { № de genótipos } \\
\text { analisados }\end{array}$ & $\begin{array}{c}\text { Porcentagem de } \\
\text { matéria seca }\end{array}$ \\
\hline SCM & 14 & $34,62 \mathrm{a}$ \\
SCC & 28 & $31,29 \mathrm{~b}$ \\
NI & 7 & $30,73 \mathrm{~b}$ \\
\hline
\end{tabular}

\footnotetext{
1 As médias seguidas pela mesma letra não diferem entre si pelo teste de Duncan $(\alpha=0,05)$.
}

e 4). A maior média foi obtida em clones da subespécie S. commersonii malmeanum $(34,62 \%)$, que diferiu estatisticamente das observadas em S. commersonii commersonii $(31,29 \%)$, e nos clones não-identificados (30,73\%), a 5\%.

Quinze bandas anódicas, com mobilidades relativas de 1,02, 1,00, 0,96, 0,92, 0,90, 0,86, 0,80, 0,77, $0,74,0,71,0,68,0,65,0,61,0,57$ e 0,51 permitiram a diferenciação de 12 padrões isoenzimáticos de aspartato transaminase, com variação de uma a cinco bandas por padrão (Fig. 1). Segundo Gottlieb (1982), geralmente, três ou quatro isoenzimas estão associadas a essas bandas anódicas de aspartato transaminase.

Dez padrões eletroforéticos foram observados para isocitrato desidrogenase, através de sete bandas anódicas e polimórficas, de mobilidades relativas de 1,08, 1,00, 0,97, 0,94, 0,92, 0,87 e 0,74, variando de uma a três por padrão (Fig. 2). Waara et al. (1989), por meio de eletroforese horizontal em gel de amido, detectaram o mesmo número de bandas nas isoenzimas anódicas de isocitrato desidrogenase em folhas de híbridos somáticos e parentais de S. tuberosum.

Os clones foram classificados em dois grupos, de acordo com maiores ou menores teores de matéria

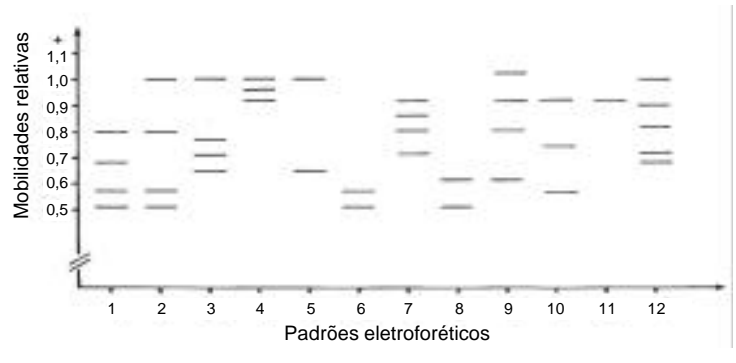

FIG. 1. Padrões eletroforéticos de aspartato transaminase: 1) clones $2,56,65,67,69$; 2) clones 139,$141 ; 3)$ clones $131,142,143$; 4) clones 25, 36, 54, 78; 5) clones 71, 119, 150, $166,168 ; 6)$ clones $7,8,55,62,113,176,196$, $199,200,222 ; 7)$ clone $108 ; 8)$ clone 53 ; 9) clones $117,118,120 ; 10)$ clones 64,109 ; 11) clone $68 ; 12$ ) clone 158. 
seca, utilizando-se, inicialmente, o critério de separálos em função da mediana $(32,00)$ dos dados do ex-

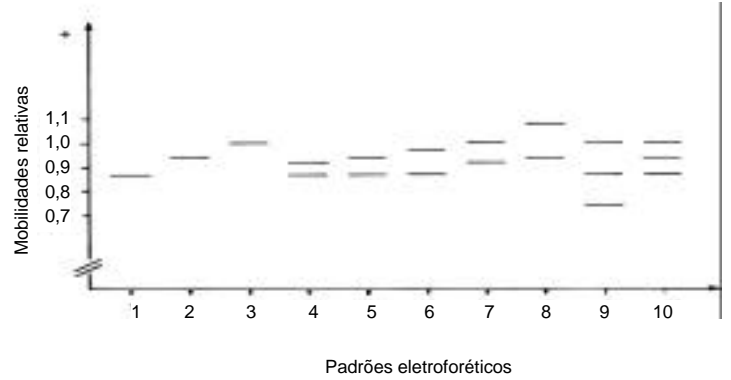

FIG. 2. Padrões eletroforéticos de isocitrato desidrogenase: 1) clones 55, 56, 64, 67, 69, 108, 109, 113,$176 ; 2$ ) clones 68,158 ; 3 ) clones 71,131 , $142,143,166 ; 4)$ clones $2,25,62,199 ; 5)$ clones 168, 196, 200, 222; 6) clones $7,8,53,65$; 7) clones 36, 54, 117, 118, 119, 120; 8) clone 78; 9) clone 150; 10) clones 139, 141. perimento. Posteriormente, verificou-se que alguns valores próximos à mediana não diferiam entre si (teste de Duncan, $\alpha=0,05)$. Estabeleceu-se, então, uma faixa de valores mais discriminatória dos dois grupos, ou seja, valores iguais ou maiores de 32,43 , em relação aos clones que apresentaram maiores teores, e os iguais ou menores que 31,56, em relação aos que continham menores teores de matéria seca. As médias de 32,43 e 31,56 diferem entre si e se encontram eqüidistantes da mediana calculada.

A associação dos grupos de clones com a banda de mobilidade relativa 1,00 de aspartato transaminase apresentou um valor de $\chi^{2}=14,6$, altamente significativo $\left(\chi_{0,05 ; 1}^{2}=3,84 ; \chi_{0,01 ; 1}^{2}=6,64\right)$ (Tabela 5). No caso da associação dos clones com a banda de mobilidade 1,00 de isocitrato desidrogenase, o valor obtido foi $\chi^{2}=7,7$, também altamente significativo (Tabela 6).

TABELA 5. Número de clones de Solanum spp. com teores de matéria seca iguais ou superiores a $32,43 \%$ ou iguais ou inferiores a $31,56 \%$, na presença ou ausência da banda de mobilidade relativa 1,00 de aspartato transaminase.

\begin{tabular}{|c|c|c|c|c|c|}
\hline \multirow{3}{*}{$\begin{array}{l}\text { Teor de matéria seca } \\
\text { dos clones }\end{array}$} & \multicolumn{4}{|c|}{ Número de clones } & \multirow[t]{3}{*}{ Total } \\
\hline & \multicolumn{2}{|c|}{ Presença da banda } & \multicolumn{2}{|c|}{ Ausência da banda } & \\
\hline & Observado & Esperado & Observado & Esperado & \\
\hline Matéria seca $\geq 32,43$ & 1 & 7 & 17 & 11 & 18 \\
\hline Matéria seca $\leq 31,56$ & 13 & 7 & 5 & 11 & 18 \\
\hline Total & 14 & 14 & 22 & 22 & 36 \\
\hline
\end{tabular}

TABELA 6. Número de clones de Solanum spp. com teores de matéria seca iguais ou superiores a $32,43 \%$ ou iguais ou inferiores a $31,56 \%$, na presença ou ausência da banda de mobilidade relativa 1,00 de isocitrato desidrogenase.

\begin{tabular}{|c|c|c|c|c|c|}
\hline \multirow{3}{*}{$\begin{array}{l}\text { Teor de matéria seca } \\
\text { dos clones }\end{array}$} & \multicolumn{4}{|c|}{ Número de clones } & \multirow[t]{3}{*}{ Total } \\
\hline & \multicolumn{2}{|c|}{ Presença da banda } & \multicolumn{2}{|c|}{ Ausência da banda } & \\
\hline & Observado & Esperado & Observado & Esperado & \\
\hline Matéria seca $\geq 32,43$ & 2 & 6,5 & 16 & 11,5 & 18 \\
\hline Matéria seca $\leq 31,56$ & 11 & 6,5 & 7 & 11,5 & 18 \\
\hline Total & 13 & 13,0 & 23 & 23,0 & 36 \\
\hline
\end{tabular}




\section{CONCLUSÃO}

Existe associação significativa entre o teor de matéria seca e as bandas de mobilidade relativa 1,00 de aspartato transaminase e de isocitrato desidrogenase; esses resultados possibilitam importantes seleções precoces de clones de batata com alto teor de matéria seca para utilização em futuros trabalhos de melhoramento.

\section{AGRADECIMENTOS}

Ao CNPq, pela concessão de bolsas; ao pesquisador Delorge Mota da Costa, responsável pela coleta e manutenção dos clones de batata silvestre, pelo fornecimento de folhas e tubérculos para análise; a Ema Gladis Schultz Corrêa e Claiton Amaral Kuhn, pelo apoio técnico.

\section{REFERÊNCIAS}

AKELEY, R.V.; MILLS, W.R.; CUNNINGHAM, C.E.; WATTS, J. Lenape: a new potato variety high in solids and chipping quality. American Potato Journal, Orono, v.45, n.4, p.142-145, 1968.

ALMEIDA, L.A.S.B. de; GASPARINO FILHO, J.; PASCHOALINO, J.E.; BENBERNHARDT, L.W.; CANTO, W.L. do. Batata pré-frita e hortaliças congeladas: economia e industrialização. São Paulo : Instituto de Tecnologia de Alimentos, 1983. 90p. (ITAL. Estudos Econômicos. Alimentos Processados, 18).

AYALA, F.J.; POWELL, J.R.; TRACEY, M.L.; MOURÃO, C.A.; PÉREZ-SALAS, S. Enzyme variability in the Drosophila willistoni group. IV. Genic variation in natural populations of Drosophila willistoni. Genetics, Bethesda, v.70, p.113-139, 1972.

COSTA, D.M.; PEREIRA, A. da S. Criação de cultivares de batata (Solanum tuberosum L.) para mesa e processamento. In: REUNIÃO ANUAL DE PESQUISA COM BATATA EM SANTA CATARINA E NO RIO GRANDE DO SUL, 2., 1995, Araranguá. Relatórios. Pelotas : Embrapa-CPACT, 1996. p.8-10.

DOUCHES, D.S.; LUDLAM, K. Electrophoretic characterization of North American potato cultivars. American Potato Journal, Orono, v.68, p.767-780, 1991.
DOUCHES, D.; SCHROETER, B.; LUDLAM, K.; HICKS, K. Allelic diversity among the Solanum species, sect Petota. American Potato Journal, Orono, v.66, n.8, p.517, 1989.

GOTTLIEB, L.D. Conservation and duplication of isozymes in plants. Science, Washington, v.216, p.373-380, 1982.

GOULD, W.A. Quality of potatoes for chip manufacture. In: SYMPOSIUM ON POTATO QUALITY INDUSTRY NEEDS FOR GROWTH, 1988, Fort Collins. Proceedings. Fort Collins : The Potato Association of America, 1988. p.10-20.

HAWKES, J.G.; HJERTING, J.P. The potatoes of Argentina, Brasil, Paraguai and Uruguay. Oxford : Claredon, 1969. 525p.

MEDINA-FILHO, H.P. Eletroforese em gel de amido: aplicações em genética e melhoramento de plantas. Campinas : Instituto Agronômico, 1983. 15p. (IAC. Circular, 121).

MENDOZA, H.A. Mejoramiento poblacional: una estrategia para la utilización del germoplasma de papa cultivada primitiva y especies silvestres. In: HIDALGO, O.A.; RINCÓN, H.R. (Ed.). Avances en el mejoramiento genético de la papa en los países del cono sur. Lima : Centro Internacional de la Papa, 1990. p.47-62.

NICHOLS, E.A.; RUDDLE, F.H. Review of enzyme polymorphism, linkage and electrophoretic conditions for mouse and somatic all hybrids in starch gels. Journal of Histochemistry and Cytochemistry, Seattle, v.21, n.12, p.1066-1081, 1973.

OLIVER, J.L.; MARTINEZ-ZAPATER, J.M. A genetic classification of potato cultivars based on allozyme patterns. Theoretical and Applied Genetics, Berlin, v.69, p.305-311, 1985.

POPE, L.R.; BEDFORD, C.L.; THOMPSON, N.R. Processing characteristics of Solanum tuberosum S. stoloniferum hybrids. American Potato Journal, Orono, v.48, p.403-409, 1971.

SCANDALIOS, J.G. Genetic control of multiple molecular forms of enzymes in plants: a review. Biochemical Genetics, New York, v.3, p.37-79, 1969.

SHIELDS, C.R.; ORTON, T.J.; STUBER, C.W. An outline of general resource needs and procedures for the electrophoretic separation of active enzymes from 
plant tissue. In: TANKSLEY, S.D.; ORTON, T.J. (Ed.). Isozymes in plant genetics and breeding. Amsterdam : Elsevier, 1983. part A, p.443-468.

SILVA, A.C.F. da; SOUZA, Z.S.; MÜLLER, J.V.; VIZZOTTO, V.J.; REBELO, J.A.; ZANINI NETO, J.A.; COSTA, D.M. da; BERTOCINI, O. EPAGRI 361 - Catucha: nova cultivar de batata, especial para fritar. Horticultura Brasileira, Brasília, v.14, n.1, p.61-62, 1996.

SOLLER, M.; PLOTKIN-HAZAN, J. The use of marker alleles for the introgression of linked quantitative alleles. Theoretical and Applied Genetics, Berlin, v.51, p.133-137, 1977.

TANKSLEY, S.D.; MEDINA-FILHO, H.; RICK, C.M. The effect of isozyme selection on metric characters in an interspecific backcross of tomato: basis of an early screening procedure. Theoretical and Applied Genetics, Berlin, v.60, p.291-296, 1981.

TANKSLEY, S.D.; MEDINA-FILHO, H.; RICK, C.M. Use of naturally occurring enzyme variation to detect and map genes controlling quantitative traits in an interspecific backcross of tomato. Heredity, Oxford, v.49, n.1, p.11-25, 1982.

VALLEJOS, C.E. Enzyme activity staining. In: TANKSLEY, S.D.; ORTON, T.J. (Ed.). Isozymes in plant genetics and breeding. Amsterdam : Elsevier, 1983. Part A, p.469-515.

WAARA, S.; TEGELSTRÖM, H.; WALLIM, A.; ERICKSSON, T. Somatic hybridization between anther derived dihaploid clones of potato (Solanum tuberosum L.) and the identification of hybrid plant by isozyme analysis. Theoretical and Applied Genetics, Berlin, v.77, p.49-56, 1989. 